\title{
Some properties of generalized $(s, k)$-Bessel function in two variables
}

\author{
R. S. Alia , S. Mubeen ${ }^{\mathrm{a}}$, K. S. Nisar ${ }^{\mathrm{b}}$, S. Araci ${ }^{\mathrm{c}, *}$, G. Rahman ${ }^{\mathrm{d}}$ \\ a Department of Mathematics, University of Sargodha, Sargodha, Pakistan. \\ ${ }^{b}$ Department of Mathematics, College of Arts and Sciences, Wadi Aldawaser, 11991, Prince Sattam bin Abdulaziz University, Kingdom \\ of Saudi Arabia. \\ ${ }^{c}$ Department of Economics, Faculty of Economics, Administrative and Social Sciences, Hasan Kalyoncu University, TR-27410 \\ Gaziantep, Turkey. \\ ${ }^{d}$ Department of Mathematics and Statistics, Hazara University, Mansehra, Pakistan.
}

\begin{abstract}
The devotion of this paper is to study the Bessel function of two variables in k-calculus. we discuss the generating function of $k$-Bessel function in two variables and develop its relations. After this we introduce the generalized ( $\mathrm{s}, \mathrm{k})$-Bessel function of two variables which help to develop its generating function. The s-analogy of k-Bessel function in two variables is also discussed. Some recurrence relations of the generalized $(s, k)$-Bessel function in two variables are also derived.
\end{abstract}

Keywords: k-Bessel function, generalized $(s, k)$-Bessel function, generalized $(s, k)$-Bessel function in two variables. 2020 MSC: 33C10, 33C50.

(C)2022 All rights reserved.

\section{Introduction}

Many special functions of mathematical physics have been generalized to a base $s$ which are known as special s-functions. The Bessel s-function is one of the essential special s-functions which was introduced by Jackson and Swarthrow [33]. Special functions in term of $k$ were presented by Diaz and Parigaun [2]. Later on, the researchers introduced various types of $k$-special functions by following the idea of Diaz and Parigaun [2]. Kokologiannaki [12] investigated further properties of k-gamma, k-beta and k-zeta functions. Mansour [15] introduced the k-generalized gamma function by functional equation. Krasniqi [13] investigated limits for k-gamma and k-beta functions. Merovci [16] gave the power product inequalities for the k-gamma function. Mubeen and Habibullah [17] proposed the so-called k-fractional integral based on gamma k-function and its applications. In [18], Mubeen and Habibullah defined the integral representation of generalized confluent hypergeometric k-functions and hypergeometric k-functions by

\footnotetext{
*Corresponding author

Email addresses: rsafdar0@gmail.com (R. S. Ali), smjhanda@gmail.com (S. Mubeen), ksnisar1@gmail.com, n.sooppy@psau.edu.sa (K. S. Nisar), serkan.araci@hku.edu.tr (S. Araci), gauhar55uom@gmail.com (G. Rahman)

doi: $10.22436 /$ jmcs.024.01.02
}

Received: 2020-04-21 Revised: 2020-05-01 Accepted: 2020-05-13 
utilizing the properties of Pochhammer k-symbols, k-gamma, and k-beta functions. In [17], Mubeen et al. proposed the following second order linear differential equation for hypergergeometric $k$-functions as

$$
k \omega(1-k x) \omega^{\prime \prime}+[\gamma-(\alpha+\beta+k) k x] \omega^{\prime}-\alpha \beta \omega=0 .
$$

The solution in the form of the so-called k-hypergeometric series of k-hypergeometric differential equation by utilizing the Frobenius method can be found in the work of Mubeen et al. [23, 22]. Recently, Li and Dong [14] investigated the hypergeometric series solutions for the second-order non-homogeneous khypergeometric differential equation with the polynomial term. Rahman et al. [27, 21] proposed the generalization of Wright hypergeometric k-functions and derived its various basic properties.

Furthermore, Mubeen and Iqbal [19] investigated the generalized version of Grüss-type inequalities by considering k-fractional integrals. Agarwal et al. [1] established certain Hermite-Hadamard type inequalities involving $k$-fractional integrals. Set et al. [32] proposed generalized Hermite-Hadamard type inequalities for Riemann-Liouville k-fractional integral. Östrowski type $k$-fractional integral inequalities can be found in the work of Mubeen et al. [20]. Many researchers have established further the generalized version of Riemann-Liouville $k$-fractional integrals and defined a large numbers of various inequalities via by using different kinds of generalized fractional integrals. The interesting readers may consult $[9,26,25,28]$. The Hadamard $k$-fractional integrals can be found in the work of Farid et al. [5]. In [6], Farid proposed the idea of Hadamard-type inequalities for k-fractional Riemann-Liouville integrals. In $[10,35]$, the authors have introduced inequalities by employing Hadamard-type inequalities for $k$-fractional integrals. Nisar et al. [24] investigated Gronwall type inequalities by utilizing RiemannLiouville k- and Hadamard k-fractional derivatives [24]. In [24], they presented dependence solutions of certain k-fractional differential equations of arbitrary real order with initial conditions. Samraiz et al. [31] proposed Hadamard k-fractional derivative and properties. Recently, Rahman et al. [29] defined generalized $k$-fractional derivative operator. Diaz and Teruel introduced the generalized gamma and beta $(\mathrm{s}, \mathrm{k})$-functions in 2005 [3]. They also proved various identities of gamma and beta $(\mathrm{s}, \mathrm{k})$-functions in two parameter deformation. In this paper, the generalized $(s, k)$-Bessel function is introduced. Firstly, the Bessel function of two variables at level $k$ is introduced by constructing its generating function and some recurrence relations. Secondly, the generating function of the generalized $(s, k)$-Bessel function is constructed and some of its recurrence relations are developed. Also, the s-analogy of the generalized $k$-Bessel function of two variables is given. Finally, the concluding comments on $(s, k)$-Bessel function are given.

\section{2. preliminaries}

In this section, we present certain well-known definition and mathematical preliminaries.

Definition 2.1 ([4]). The s-factorial is defined by

$$
[n]_{s} !=\frac{(s ; s)_{n}}{(1-s)^{n}}
$$

where $n$ is any positive integer and $0<s<1$. Replacing $n$ by $n+k$ in $(2.1)$, where $k>0$, we get

$$
[n+k]_{s} !=\frac{(s ; s)_{n+k}}{(1-s)^{n+k}}
$$

Definition $2.2([3])$. The generalized $(s, k)$-gamma function is defined as

$$
\Gamma_{s, k}(t)=\frac{\left(1-s^{k}\right)_{s, k}^{\frac{t}{k}-1}}{(1-s)^{\frac{t}{k}-1}}, \quad t>0,
$$

where $k$ is any positive real number and $0<s<1$. 
After changing of variable $t$ by $n k$, we get

$$
\Gamma_{s, k}(n k)=\prod_{j=1}^{n-1}[j k]_{s}=\prod_{j=1}^{n-1} \frac{\left(1-s^{j k}\right)}{(1-s)}=\frac{\left(1-s^{k}\right)_{s, k}^{n-1}}{(1-s)^{n-1}} .
$$

Definition 2.3 ([34]). The s-Bessel function of two variables $x$ and $y$ is given by

$$
J_{v \cdot \mu}(x, y ; s)=\sum_{m, n=0}^{\infty} \frac{(-1)^{m+n}\left(\frac{x}{2}\right)^{2 m+v}\left(\frac{y p(x)}{2}\right)^{2 n+\mu}}{[m]_{s} ![n]_{s} ! \Gamma_{s}(v+m+1) \Gamma_{s}(\mu+n+1)},
$$

where $\nu, \mu$ are not negative integers.

Definition 2.4. The relation between s-gamma function and $(s, k)$-gamma function is given by

$$
\lim _{s \rightarrow 1} \Gamma_{s, k}(n k)=\lim _{s \rightarrow 1} \Gamma_{s^{k}}(n)=k^{n-1} \Gamma(n),
$$

where $k>0,0<s<1$ and $n$ is positive real number.

\section{The k-Bessel function and generalized $(s, k)$-Bessel function in two variables}

In this section, we introduce $k$-Bessel function and generalized $(s, k)$-Bessel function in two variables.

Definition 3.1. The k-Bessel function in two variables is defined as

$$
J_{v, \mu}^{k}(x, y)=\sum_{m, n=0}^{\infty} \frac{(-1)^{m+n}\left(\frac{x}{2}\right)^{2 m+\frac{v}{k}}\left(\frac{y p(x)}{2}\right)^{2 n+\frac{\mu}{k}}}{m ! n ! \Gamma_{k}(v+m k+k) \Gamma_{k}(\mu+n k+k)} .
$$

If $\nu$ and $\mu$ are not negative integers, then we have

$$
J_{-v,-\mu}^{k}(x, y)=(-1)^{v+\mu} J_{v, \mu}^{k}(x, y) .
$$

Definition 3.2. The generalized $(s, k)$-Bessel function of two variables $x, y$ is defined by

$$
J_{v, \mu}^{k}(x, y ; s)=\sum_{m, n=0}^{\infty} \frac{(-k)^{m+n}\left(\frac{x}{2}\right)^{2 m+\frac{v}{k}\left(\frac{y p(x)}{2}\right)^{2 n+\frac{\mu}{k}}}}{[m]_{s^{k}} ![n]_{s} ! \Gamma_{s, k}(v+m k+k) \Gamma_{s, k}(\mu+n k+k)^{\prime}},
$$

where $k$ is any positive real number, $0<s<1$ and $\nu, \mu$ are non negative integers.

Remark 3.3. If we let $k=1$, then the generalized $(s, k)$-Bessel function reduces to $s$-Bessel function (2.3).

Remark 3.4. If we let $s=1$, then the generalized $(s, k)$-Bessel function reduces to k-Bessel function (3.1).

Remark 3.5. If we let $s=k=1$, then the generalized $(s, k)$-Bessel function reduces to the following Bessel function in two variables

$$
J v, \mu(x, y)=\sum_{m, n=0}^{\infty} \frac{(-1)^{m+n}\left(\frac{x}{2}\right)^{2 m+v}\left(\frac{y p(x)}{2}\right)^{2 n+\mu}}{m ! n ! \Gamma(v+m+1) \Gamma(\mu+n+1)}
$$

where $\nu, \mu$ are non negative integers.

\section{Properties of Bessel s-function and Bessel $(s, k)$-function in two variables}

The study of Bessel function and s-Bessel function of two variables in k-calculus gives important theories in the filed of analysis. We discuss some important results about $k$-Bessel function and (s, $k$ )-Bessel function in two variables. We derive the generating function of k-Bessel function of two variables, and also discuss the s-analogy of the generalized k-Bessel function of two variables in the form of theorems. 
Lemma 4.1. The relation between Bessel function and $\mathrm{k}$-Bessel function in two variables is given by

$$
J_{v, \mu}^{k}(x, y)=k^{\frac{-(v+\mu)}{2 k}} J_{\frac{v}{k}, \frac{\mu}{k}}\left(\frac{x}{\sqrt{k}}, \frac{y}{\sqrt{k}}\right)
$$

or counter part is

$$
J_{\frac{v}{k}, \frac{\mu}{k}}(x, y)=k^{\frac{v+\mu}{2 k}} J_{v, \mu}^{k}(x \sqrt{k}, y \sqrt{k}),
$$

where $\nu, \mu$ are non negative integers and $k$ is any positive real number.

Proof. By definition of k-Bessel function, we have

$$
\begin{aligned}
J_{v, \mu}^{k}(x, y) & =\sum_{m, n=0}^{\infty} \frac{(-1)^{m+n}\left(\frac{x}{2}\right)^{2 m+\frac{v}{k}}\left(\frac{y p(x)}{2}\right)^{2 n+\frac{\mu}{k}}}{[m] ![n] ! \Gamma_{k}(v+m k+k) \Gamma_{k}(\mu+n k+k)} \\
& =k^{\frac{-(v+\mu)}{2 k}} \sum_{m, n=0}^{\infty} \frac{(-1)^{m+n}\left(\frac{x}{2 \sqrt{k}}\right)^{2 m+\frac{v}{k}}\left(\frac{y p(x)}{2 \sqrt{k}}\right)^{2 n+\frac{\mu}{k}}}{[m] ![n] ! \Gamma\left(\frac{v}{k}+m+1\right) \Gamma\left(\frac{\mu}{k}+n+1\right)}=k^{\frac{-(v+\mu)}{2 k}} J_{\frac{v}{k}, \frac{\mu}{k}}\left(\frac{x}{\sqrt{k}}, \frac{y}{\sqrt{k}}\right) .
\end{aligned}
$$

After replacing $x$ by $x \sqrt{k}$ and $y$ by $y \sqrt{k}$ in equation (4.3), we get (4.2).

Lemma 4.2. The following relation holds for Bessel function and k-Bessel function of two variables

$$
J_{v k, \mu k}^{k}(x, y)=k^{\frac{-(v+\mu)}{2}} J_{v, \mu}\left(\frac{x}{\sqrt{k}}, \frac{y}{\sqrt{k}}\right)
$$

or its counter part is

$$
J_{v, \mu}(x, y)=k^{\frac{v+\mu}{2}} J_{v k, \mu k}^{k}(x \sqrt{k}, y \sqrt{k}),
$$

where $\nu, \mu$ are integers and $k$ is any positive real number.

Proof. Consider the definition of k-Bessel function in two variables, we have

$$
J_{v, \mu}^{k}(x, y)=\sum_{m, n=0}^{\infty} \frac{(-1)^{m+n}\left(\frac{x}{2}\right)^{2 m+\frac{v}{k}}\left(\frac{y p(x)}{2}\right)^{2 n+\frac{\mu}{k}}}{[m] ![n] ! \Gamma_{k}(v+m k+k) \Gamma_{k}(\mu+n k+k)} .
$$

After replacing $\nu$ by $v k$ and $\mu$ by $\mu k$ in equation (4.4), we have

$$
\begin{aligned}
J_{v k, \mu k}^{k}(x, y) & =\sum_{m, n=0}^{\infty} \frac{(-1)^{m+n}\left(\frac{x}{2}\right)^{2 m+v}\left(\frac{y p(x)}{2}\right)^{2 n+\mu}}{[m] ![n] ! \Gamma_{k}(v k+m k+k) \Gamma_{k}(\mu k+n k+k)} . \\
& =k^{\frac{-(v+\mu)}{2}} \sum_{m, n=0}^{\infty} \frac{(-1)^{m+n}\left(\frac{x}{2 \sqrt{k}}\right)^{2 m+v}\left(\frac{y p(x)}{2 \sqrt{k}}\right)^{2 n+\mu}}{[m] ![n] ! \Gamma(v+m+1) \Gamma(\mu+n+1)}=k^{\frac{-(v+\mu)}{2}} J_{v, \mu}\left(\frac{x}{\sqrt{k}}, \frac{y}{\sqrt{k}}\right) .
\end{aligned}
$$

After replacing $x$ by $x \sqrt{k}$ and $y$ by $y \sqrt{k}$ in equation (4.5), we have

$$
J_{v k, \mu k}^{k}(x \sqrt{k}, y \sqrt{k})=k^{\frac{-(v+\mu)}{2}} J_{v, \mu}(x, y) \quad \text { or } \quad J_{v, \mu}(x, y)=k^{\frac{(v+\mu)}{2}} J_{v k, \mu k}^{k}(x \sqrt{k}, y \sqrt{k}) .
$$

Lemma 4.3. The k-Bessel function in two variables satisfies

$$
J_{-v,-\mu}^{k}(x, y)=(-k)^{\frac{v+\mu}{k}} J_{\nu, \mu}^{k}(x, y),
$$

where $\nu, \mu$ are non negative integers and $k$ is any positive real number. 
Proof. Replacing the value of $\nu, \mu$ by $-v,-\mu$ in equation (4.1), and resulting equation is as follows

$$
J_{-v,-\mu}^{k}(x, y)=k^{\frac{v+\mu}{2 k}} J_{-\frac{v}{k},-\frac{\mu}{k}}\left(\frac{x}{\sqrt{k}}, \frac{y}{\sqrt{k}}\right) .
$$

Using the value of the equation (3.2), we get

$$
J_{-v,-\mu}^{k}(x, y)=(-1)^{\frac{v+\mu}{k}} k^{\frac{v+\mu}{2 k}} J_{\frac{v}{k}, \frac{\mu}{k}}\left(\frac{x}{\sqrt{k}}, \frac{y}{\sqrt{k}}\right)=(-k)^{\frac{v+\mu}{2 k}} k^{-\frac{(v+\mu)}{2 k}} J_{\frac{v}{k}, \frac{\mu}{k}}\left(\frac{x}{\sqrt{k}}, \frac{y}{\sqrt{k}}\right)=(-k)^{\frac{v+\mu}{k}} J_{v, \mu}^{k}(x, y) .
$$

Theorem 4.4. For $\mathrm{t} \neq 0, w \neq 0$ and $\mathrm{t}, w \in \mathbb{C}$, then the generating function of $\mathrm{k}$-Bessel function in two variables is

$$
\exp \left[\frac{x}{2 \sqrt{k}}\left(\frac{t}{\sqrt{k}}-\frac{\sqrt{k}}{t}\right)+\frac{y p(x)}{2 \sqrt{k}}\left(\frac{w}{\sqrt{k}}-\frac{\sqrt{k}}{w}\right)\right]=\sum_{v, \mu=-\infty}^{\infty} t^{v} w^{\mu} J_{v k, \mu k}^{k}(x, y),
$$

where $\nu, \mu$ are non negative integers and $\mathrm{k}$ is any positive real number.

Proof. Let

$$
A \equiv \sum_{\nu, \mu=-\infty}^{\infty} t^{v} w^{\mu} J_{v k, \mu k}^{k}(x, y), \equiv \sum_{\nu, \mu=-\infty}^{-1} t^{v} w^{\mu} J_{v k, \mu k}^{k}(x, y)+\sum_{\nu, \mu=0}^{\infty} t^{v} w^{\mu} J_{v k, \mu k}^{k}(x, y) .
$$

After replacing $v$ by $-v-1$ and $\mu$ by $-\mu-1$ in first summation of the equation (4.7), we have

$$
A \equiv \sum_{\nu, \mu=0}^{\infty} t^{-v-1} w^{-\mu-1} J_{-(v+1) k,-(\mu+1) k}^{k}(x, y)+\sum_{\nu, \mu=0}^{\infty} t^{v} w^{\mu} J_{v k, \mu k}^{k}(x, y) .
$$

By using equation (4.6) in equation (4.8), we have

$$
\begin{aligned}
A \equiv & \sum_{\nu, \mu=0}^{\infty} t^{-v-1} w^{-\mu-1}(-k)^{v+\mu+2} J_{(v+1) k,(\mu+1) k}^{k}(x, y)+\sum_{\nu, \mu=0}^{\infty} t^{v} w^{\mu} J_{v k, \mu k}^{k}(x, y) \\
\equiv & \sum_{\nu, \mu=0}^{\infty} \sum_{m, n=0}^{\infty} t^{-v-1} w^{-\mu-1}(-k)^{v+\mu+2} \frac{(-1)^{m+n}\left(\frac{x}{2}\right)^{2 m+v+1}\left(\frac{y p(x)}{2}\right)^{2 n+\mu+1}}{m ! n ! \Gamma_{k}(m k+(v+1) k+k) \Gamma_{k}(n k+(\mu+1) k+k)} \\
& +\sum_{\nu, \mu=0}^{\infty} \sum_{m, n=0}^{\infty} \frac{t^{\nu} w^{\mu}(-1)^{m+n}\left(\frac{x}{2}\right)^{2 m+v}\left(\frac{y p(x)}{2}\right)^{2 n+\mu}}{m ! n ! \Gamma_{k}(m k+v k+k) \Gamma_{k}(n k+\mu k+k)} .
\end{aligned}
$$

After replacing $v$ by $v-2 m$ and $\mu$ by $\mu-2 n$ in the equation (4.9), we have

$$
\begin{aligned}
A \equiv & \sum_{\nu=0}^{\infty} \sum_{m=0}^{\frac{v}{2}} \frac{t^{-v-2 m-1}(-1)^{v-m+1} k^{v-2 m+1}\left(\frac{x}{2}\right)^{v+1}}{m ! \Gamma_{k}(m k+(v-2 m) k+2 k)} \\
& +\sum_{\mu=0}^{\infty} \sum_{n=0}^{\frac{\mu}{2}} \frac{w^{-\mu-2 n-1}(-1)^{\mu-n+1} k^{\mu-2 n+1}\left(\frac{y p(x)}{2}\right)^{\mu+1}}{n ! \Gamma_{k}(n k+(v-2 n) k+2 k)} \\
& +\sum_{v=0}^{\infty} \sum_{m=0}^{\frac{v}{2}} \frac{t^{v-2 m}(-1)^{m}\left(\frac{x}{2}\right)^{v}}{m ! \Gamma_{k}(m k+(v-2 m) k+2 k)}+\sum_{\mu=0}^{\infty} \sum_{n=0}^{\frac{\mu}{2}} \frac{w^{\mu-2 n}(-1)^{n}\left(\frac{y p(x)}{2}\right)^{\mu}}{n ! \Gamma_{k}(n k+(\mu-2 n) k+2 k)}
\end{aligned}
$$


After replacing $v$ by $v-1$ and $\mu$ by $\mu-1$ in first and second summation of the equation (4.10), we have

$$
\begin{aligned}
A \equiv & \sum_{v=1}^{\infty} \sum_{m=0}^{\frac{v-1}{2}} \frac{t^{-v+2 m}(-1)^{v-m} k^{v-2 m}\left(\frac{x}{2}\right)^{v}}{m ! \Gamma_{k}(v k-m k+k)}+\sum_{\mu=1}^{\infty} \sum_{n=0}^{\frac{\mu-1}{2}} \frac{w^{-\mu+2 n}(-1)^{\mu-n} k^{\mu-2 n}\left(\frac{y p(x)}{2}\right)^{\mu}}{n ! \Gamma_{k}(\mu k-n k+k)}+2 \\
& +\sum_{v=1}^{\infty} \sum_{m=0}^{\frac{v}{2}} \frac{t^{v-2 m}(-1)^{m}\left(\frac{x}{2}\right)^{v}}{m ! \Gamma_{k}(v k-m k+k)}+\sum_{\mu=1}^{\infty} \sum_{n=0}^{\frac{\mu}{2}} \frac{w^{\mu-2 n}(-1)^{n}\left(\frac{y p(x)}{2}\right)^{\mu}}{n ! \Gamma_{k}(\mu k-n k+k)} \\
\equiv & \sum_{v=1}^{\infty} \sum_{m=0}^{\frac{v-1}{2}} \frac{t^{-v+2 m}(-1)^{v-m} k^{-m}\left(\frac{x}{2}\right)^{v}}{m ! \Gamma(v-m+1)}+\sum_{\mu=1}^{\infty} \sum_{n=0}^{\frac{\mu-1}{2}} \frac{w^{-\mu+2 n}(-1)^{\mu-n} k^{-n}\left(\frac{y p(x)}{2}\right)^{\mu}}{n ! \Gamma(\mu-n+1)} \\
& +2+\sum_{v=1}^{\infty} \sum_{m=0}^{\frac{v}{2}} \frac{t^{v-2 m}(-1)^{m} k^{-v+m}\left(\frac{x}{2}\right)^{v}}{m ! \Gamma(v-m+1)}+\sum_{\mu=1}^{\infty} \sum_{n=0}^{\frac{\mu}{2}} \frac{w^{\mu-2 n}(-1)^{n} k^{-\mu+n}\left(\frac{y p(x)}{2}\right)^{\mu}}{n ! \Gamma(\mu-n+1)} .
\end{aligned}
$$

By rearranging the terms, we have

$$
\begin{aligned}
A \equiv & 2+\sum_{\nu=1}^{\infty} \sum_{m=0}^{\frac{v}{2}} \frac{(-1)^{m}\left(\frac{t}{\sqrt{k}}\right)^{v-m-m}\left(\frac{x}{2 \sqrt{k}}\right)^{\nu}}{m !(v-m) !}+\sum_{\mu=1}^{\infty} \sum_{n=0}^{\frac{\mu}{2}} \frac{(-1)^{n}\left(\frac{w}{\sqrt{k}}\right)^{\mu-n-n}\left(\frac{y p(x)}{2 \sqrt{k}}\right)^{\mu}}{n !(\mu-n) !} \\
& +\sum_{\nu=1}^{\infty} \sum_{m=0}^{\frac{v-1}{2}} \frac{(-1)^{v-m}\left(\frac{t}{\sqrt{k}}\right)^{m-(v-m)}\left(\frac{x}{2 \sqrt{k}}\right)^{\nu}}{m !(v-m) !}+\sum_{\mu=1}^{\infty} \sum_{n=0}^{\frac{v-1}{2}} \frac{(-1)^{\mu-n}\left(\frac{w}{\sqrt{k}}\right)^{n-(\mu-n)}\left(\frac{y p(x)}{2 \sqrt{k}}\right)^{\mu}}{n !(\mu-n) !}
\end{aligned}
$$

By using [30, Lemma 12, page 112], we have

$$
\begin{aligned}
A & \equiv 2+\sum_{v=1}^{\infty} \sum_{m=0}^{v} \frac{(-1)^{m}\left(\frac{t}{\sqrt{k}}\right)\left(\frac{x}{2 \sqrt{k}}\right)^{v}}{m !(v-m) !}+\sum_{\mu=1}^{\infty} \sum_{n=0}^{\mu} \frac{(-1)^{n}\left(\frac{w}{\sqrt{k}}\right)^{\mu-n-n}\left(\frac{y p(x)}{2 \sqrt{k}}\right)^{\mu}}{n !(\mu-n) !} \\
& \equiv \sum_{v=0}^{\infty} \sum_{m=0}^{v} \frac{(-1)^{m}\left(\frac{t}{\sqrt{k}}\right)^{v-m}\left(\frac{\sqrt{k}}{t}\right)^{m}\left(\frac{x}{2 \sqrt{k}}\right)^{v}}{m !(v-m) !}+\sum_{\mu=0}^{\infty} \sum_{n=0}^{\mu} \frac{(-1)^{n}\left(\frac{w}{\sqrt{k}}\right)^{\mu-n}\left(\frac{\sqrt{k}}{w}\right)^{n}\left(\frac{y p(x)}{2 \sqrt{k}}\right)^{\mu}}{n !(\mu-n) !} \\
& \equiv \sum_{v=0}^{\infty} \frac{\left(\frac{t}{\sqrt{k}}-\frac{\sqrt{k}}{t}\right)^{v}\left(\frac{x}{2 \sqrt{k}}\right)^{v}}{m !(v) !}+\sum_{\mu=0}^{\infty} \frac{\left(\frac{w}{\sqrt{k}}-\frac{\sqrt{k}}{w}\right)^{\mu}\left(\frac{y p(x)}{2 \sqrt{k}}\right)^{\mu}}{n !(\mu) !} \\
& \equiv \exp \left[\frac{x}{2 \sqrt{k}}\left(\frac{t}{\sqrt{k}}-\frac{\sqrt{k}}{t}\right)+\frac{y p(x)}{2 \sqrt{k}}\left(\frac{w}{\sqrt{k}}-\frac{\sqrt{k}}{w}\right)\right]
\end{aligned}
$$

which is required generating function of k-Bessel function in two variables.

Lemma 4.5. The $(\mathrm{s}, \mathrm{k})$-Bessel function of two variables satisfies the relation

$$
J_{v, \mu}^{k}(-x, y ; s)=(-1)^{\frac{v}{k}} J_{v, \mu}^{k}(x, y ; s),
$$

where $v, \mu$ are integers, $k$ is any real number and $0<s<1$.

Proof. Since $(s, k)$-Bessel function in two variables is

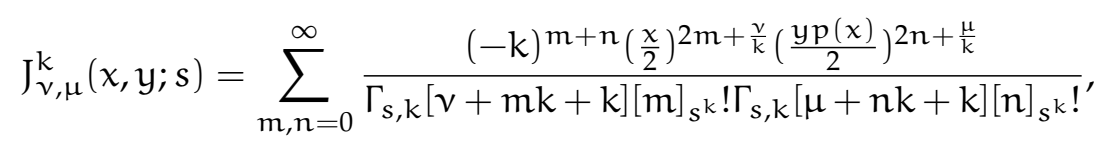


by changing $x$ by $-x$ in the above, we get

$$
\begin{aligned}
& J_{v, \mu}^{k}(-x, y ; s)=\sum_{m, n=0^{\infty}} \frac{(-k)^{m+n}\left(\frac{-x}{2}\right)^{2 m+\frac{v}{k}\left(\frac{y p(x)}{2}\right)^{2 n+\frac{\mu}{k}}}}{\Gamma_{s, k}[v+m k+k][m]_{s} !_{s, k}[\mu+n k+k][n]_{s} !} \\
& =(-1)^{2 m+\frac{v}{k}} \sum_{m, n=0}^{\infty} \frac{(-k)^{m+n}\left(\frac{x}{2}\right)^{2 m+\frac{v}{k}\left(\frac{y p(x)}{2}\right)^{2 n+\frac{\mu}{k}}}}{\Gamma_{s, k}[v+m k+k][m]_{s} ! \Gamma_{s, k}[\mu+n k+k][n]_{s^{k}} !} .
\end{aligned}
$$

Here, $(-1)^{2 m}$ is positive for all values of $m$. Therefore, $(-1)^{2 m}=1$, then we have

$$
\mathrm{J}_{v, \mu}^{k}(-x, y ; s)=(-1)^{\frac{v}{k}} \sum_{m, n=0}^{\infty} \frac{(-k)^{m+n}\left(\frac{x}{2}\right)^{2 m+\frac{v}{k}}\left(\frac{y p(x)}{2}\right)^{2 n+\frac{\mu}{k}}}{\Gamma_{s, k}[v+m k+k][m]_{s} ! \Gamma_{s, k}[\mu+n k+k][n]_{s} !}=(-1)^{\frac{v}{k}} J_{v, \mu}^{k}(x, y ; s) .
$$

Lemma 4.6. The (s, k)-Bessel function of two variables holds

$$
J_{\nu, \mu}^{k}(x,-y ; s)=(-1)^{\frac{\mu}{k}} J_{\nu, \mu}^{k}(x, y ; s),
$$

where $v, \mu$ are non negative integers, $k$ is any real positive number and $0<\mathrm{s}<1$.

Proof. The $(s, k)$-Bessel function is

$$
J_{v, \mu}^{k}(x, y ; s)=\sum_{m, n=0}^{\infty} \frac{(-k)^{m+n}\left(\frac{x}{2}\right)^{2 m+\frac{v}{k}\left(\frac{y p(x)}{2}\right)^{2 n+\frac{\mu}{k}}}}{\Gamma_{s, k}[v+m k+k][m]_{s} ! \Gamma_{s, k}[\mu+n k+k][n]_{s^{k}} !} .
$$

After replacing $y$ by $-y$ in the equation (4.11), we have

$$
\begin{aligned}
& J_{v, \mu}^{k}(x,-y ; s)=\sum_{m, n=0} \frac{(-k)^{m+n}\left(\frac{x}{2}\right)^{2 m+\frac{v}{k}}\left(\frac{-y p(x)}{2}\right)^{2 n+\frac{\mu}{k}}}{\Gamma_{s, k}[v+m k+k][m]_{s} !_{s, k}[\mu+n k+k][n]_{s^{k}} !} \\
& =(-1)^{2 n+\frac{\mu}{k}} \sum_{m, n=0}^{\infty} \frac{(-k)^{m+n}\left(\frac{x}{2}\right)^{2 m+\frac{v}{k}\left(\frac{y p(x)}{2}\right)^{2 n+\frac{\mu}{k}}}}{\Gamma_{s, k}[v+m k+k][m]_{s^{k}} ! \Gamma_{s, k}[\mu+n k+k][n]_{s^{k}} !} .
\end{aligned}
$$

For all values of $n,(-1)^{2 n}$ is positive. Therefore, $(-1)^{2 n}=1$, then we have

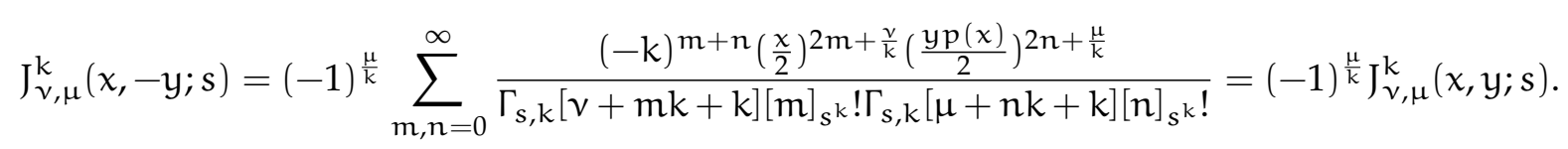

Lemma 4.7. The ( $\mathrm{s}, \mathrm{k})$-Bessel function of two variables holds

$$
\mathrm{J}_{v, \mu}^{\mathrm{k}}(-x,-y ; s)=(-1)^{\frac{v+\mu}{k}} \mathrm{~J}_{v, \mu}^{\mathrm{k}}(x, y ; s),
$$

where $v, \mu$ are non negative integers, $k$ is any real positive number and $0<s<1$.

Proof. The $(s, \mathrm{k})$-Bessel function of two variables is given by

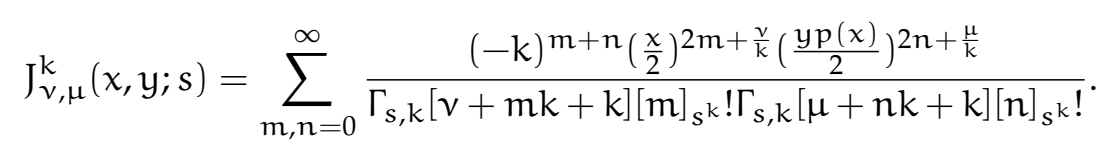


After replacing $x$ by $-x$ and $y$ by $-y$ in the above, we have

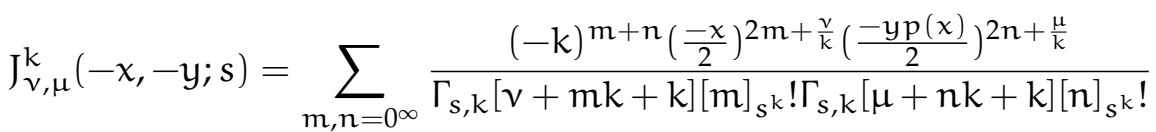

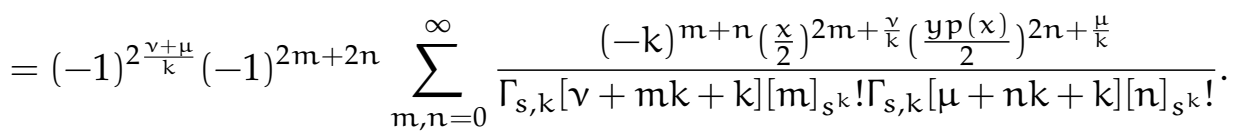

For all values of $m$ and $n,(-1)^{2 m+2 n}$ is positive. Therefore, $(-1)^{2 m+2 n}=1$, then we have

$$
J_{v, \mu}^{k}(-x,-y ; s)=(-1)^{\frac{v+\mu}{k}} \sum_{m, n=0}^{\infty} \frac{(-k)^{m+n}\left(\frac{x}{2}\right)^{2 m+\frac{v}{k}}\left(\frac{y p(x)}{2}\right)^{2 n+\frac{\mu}{k}}}{\Gamma_{s, k}[v+m k+k][m]_{s} k ! \Gamma_{s, k}[\mu+n k+k][n]_{s} k}=(-1)^{\frac{v+\mu}{k}} J_{v, \mu}^{k}(x, y ; s) .
$$

Now, we construct the generating function of the generalized $(s, k)$-Bessel function of two variables.

Theorem 4.8. Prove that the generating function of the generalized Bessel $\mathrm{q}, \mathrm{k}$-function of two variables is the expansion of

$$
E_{s^{k}}\left[\frac{x}{2}\left(t-\frac{k}{t}\right)+\frac{y p(x)}{2}\left(w-\frac{k}{w}\right)\right]
$$

where $\mathrm{t} \neq 0, w \neq 0, \mathrm{t}, w \in \mathbb{C}$, and $\mathrm{k}$ is any positive real number.

Proof. There are two important cases of exponential s-function which are defined by

$$
E_{s}(x)=\sum_{r=0}^{\infty} \frac{x^{r}}{[r]_{s} !}
$$

and

$$
E_{s}(x)=\sum_{r=0}^{\infty} \frac{s^{\frac{r^{2}}{2}} x^{r}}{(s ; s)_{r}}, \quad|x|<1 .
$$

By taking limit $s \rightarrow 1$, we get $\lim _{s \rightarrow 1}\left(E_{s}(1-s) x\right)=e^{x}$. Taking left hand side of (4.12) and using (4.13), we have

$$
E_{s^{k}}\left[\frac{x}{2}\left(t-\frac{k}{t}\right)+\frac{y p(x)}{2}\left(w-\frac{k}{w}\right)\right]=\sum_{v=0}^{\infty} \frac{\left(\frac{x t}{2}\right)^{v}}{[v]_{s^{k}} !} \sum_{m=0}^{\infty} \frac{\left(\frac{-k x}{2 t}\right)^{m}}{[m]_{s^{k}} !} \sum_{\mu=0}^{\infty} \frac{\left(\frac{y p(x) w}{2}\right)^{\mu}}{[\mu]_{s^{k}} !} \sum_{n=0}^{\infty} \frac{\left(\frac{-k y p(x)}{2 w}\right)^{n}}{[n]_{s^{k}} !} .
$$

Replacing $\nu$ by $\frac{v}{k}+m$ and $\mu$ by $\frac{\mu}{k}+n$ in the equation (4.14), we have

$$
\begin{aligned}
& =\sum_{v=-\infty}^{\infty} \frac{\left(\frac{x t}{2}\right)^{\frac{v}{k}}+m}{\left[\frac{v}{k}+m\right]_{q^{k}} !} \sum_{m=0}^{\infty} \frac{\left(\frac{-k x}{2 t}\right)^{m}}{[m]_{s^{k}} !} \sum_{\mu=-\infty}^{\infty} \frac{\left(\frac{y p(x) w}{2}\right)^{\frac{\mu}{k}+n}}{\left[\frac{\mu}{k}+n\right]_{s^{k}} !} \sum_{n=0}^{\infty} \frac{\left(\frac{-k y p(x)}{2 w}\right)^{n}}{[n]_{s^{k}} !} \\
& =\sum_{\nu, \mu=-\infty}^{\infty} \sum_{m, n=0}^{\infty} \frac{(-k)^{m}\left(\frac{x}{2}\right)^{\frac{v}{k}+2 m} t^{\frac{v}{k}+m-m}\left(\frac{y p(x)}{2}\right)^{\frac{\mu}{k}+2 n}(-k)^{n} w^{\frac{\mu}{k}+n-n}}{\Gamma_{s^{k}}\left[\frac{v}{k}+m+1\right][m]_{s^{k}} ! \Gamma_{q^{k}}\left[\frac{\mu}{k}+n+1\right][n]_{s^{k}} !}
\end{aligned}
$$

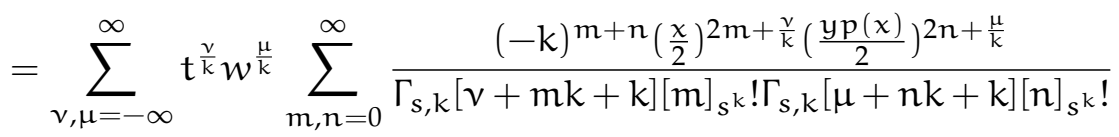

$$
\begin{aligned}
& =\sum_{\nu, \mu=-\infty}^{\infty} t^{\frac{v}{k}} \mathcal{W}^{\frac{\mu}{k}} J_{\nu, \mu}^{k}(x, y ; s),
\end{aligned}
$$

which is required generating function for $(s, k)$-Bessel function of two variables. 
Lemma 4.9. If the parameters $v$ and $\mu$ are integers then generalized $(s, k)$-Bessel function satisfies

$$
J_{-v, \mu}^{k}(x, y ; s)=(-k)^{\frac{v}{k}} J_{v, \mu}^{k}(x, y ; s) .
$$

Proof. Replacing $v$ by $-v$ in $(s, k)$-Bessel function of two variables we get

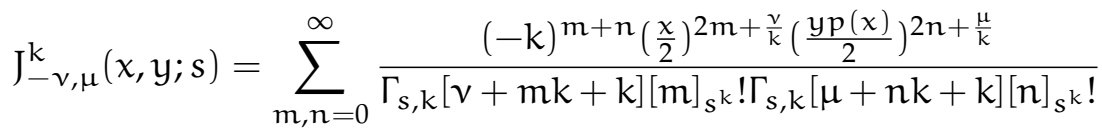

$$
\begin{aligned}
& =\sum_{m, n=0}^{\infty} \frac{(-k)^{m+n}\left(\frac{x}{2}\right)^{2 m+\frac{v}{k}}\left(\frac{y p(x)}{2}\right)^{2 n+\frac{\mu}{k}}}{\Gamma_{s^{k}}\left[\frac{v}{k}+m+1\right][m]_{s} ! \Gamma_{s, k}[\mu+n k+k][n]_{s^{k}} !} \text {. }
\end{aligned}
$$

Substituting $v$ by $-v$ in the equation (4.15), we get

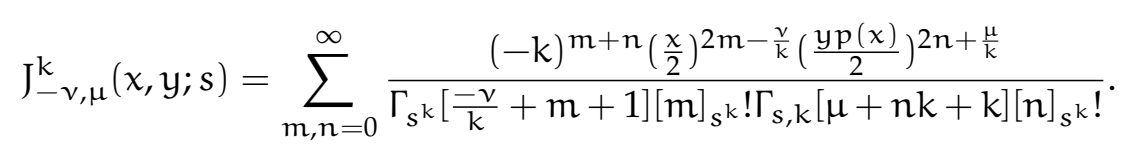

After replacing $m$ by $\frac{v}{k}+r$ in equation (4.16), we have

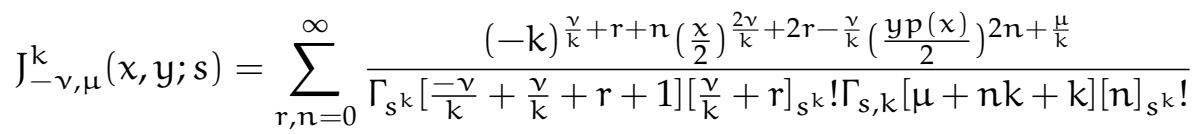

$$
\begin{aligned}
& =\sum_{r, n=0}^{\infty} \frac{(-k)^{\frac{v}{k}}(-k)^{r+n}\left(\frac{x}{2}\right)^{2 r+\frac{v}{k}}\left(\frac{y p(x)}{2}\right)^{2 n+\frac{\mu}{k}}}{\Gamma_{s^{k}}[r+1] \Gamma_{s^{k}}\left[\frac{v}{k}+r+1\right] \Gamma_{s, k}[\mu+n k+k][n]_{s^{k}} !} \\
& =(-k)^{\frac{v}{k}} \sum_{r, n=0}^{\infty} \frac{(-k)^{r+n}\left(\frac{x}{2}\right)^{2 r+\frac{v}{k}}\left(\frac{y p(x)}{2}\right)^{2 n+\frac{\mu}{k}}}{\Gamma_{s, k}[v+r k+k][r]_{s} ! \Gamma_{s, k}[\mu+n k+k][n]_{s} k}=(-k)^{\frac{v}{k}} J_{v, \mu}^{k}(x, y ; s),
\end{aligned}
$$

which is required recurrence relation.

Lemma 4.10. If the parameters $v$ and $\mu$ are integers, then $(s, k)$-Bessel function of two variables satisfies the relation

$$
J_{v,-\mu}^{k}(x, y ; s)=(-k)^{\frac{\mu}{k}} J_{v, \mu}^{k}(x, y ; s) .
$$

Proof. Consider the $(\mathrm{s}, \mathrm{k})$-Bessel function of two variables

$$
\begin{aligned}
& J_{v, \mu}^{k}(x, y ; s)=\sum_{m, n=0}^{\infty} \frac{(-k)^{m+n}\left(\frac{x}{2}\right)^{2 m+\frac{v}{k}\left(\frac{y p(x)}{2}\right)^{2 n+\frac{\mu}{k}}}}{\Gamma_{s, k}[v+m k+k][m]_{s} !_{s, k}[v+n k+k][n]_{s} k !} \\
& =\sum_{m, n=0}^{\infty} \frac{(-k)^{m+n}\left(\frac{x}{2}\right)^{2 m+\frac{v}{k}}\left(\frac{y p(x)}{2}\right)^{2 n+\frac{\mu}{k}}}{\Gamma_{s, k}[v+m k+k][m]_{s} !\left[n+\frac{\mu}{k}\right]_{s^{k}} ![n]_{s^{k}} !} \text {. }
\end{aligned}
$$

Replacing $\mu$ by $-\mu$ in equation (4.17), we have

$$
J_{v,-\mu}^{k}(x, y ; s)=\sum_{m, n=0}^{\infty} \frac{(-k)^{m+n}\left(\frac{x}{2}\right)^{2 m+\frac{v}{k}\left(\frac{y p(x)}{2}\right)^{2 n-\frac{\mu}{k}}}}{\Gamma_{s, k}[v+m k+k][m]_{s^{k}} !\left[n-\frac{\mu}{k}\right]_{s^{k}} ![n]_{s^{k}} !} .
$$

Replacing $n$ by $s+\frac{\mu}{k}$ in equation (4.18), we get

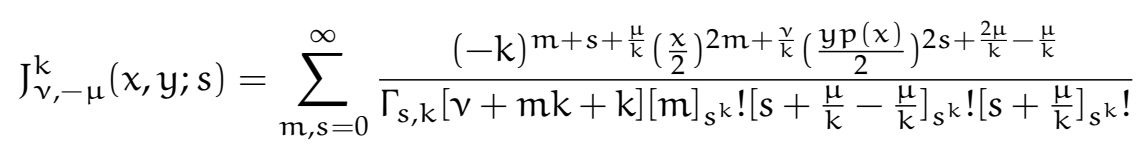




$$
\begin{aligned}
& =(-k)^{\frac{\mu}{k}} \sum_{m, s=0}^{\infty} \frac{(-k)^{m+s}\left(\frac{x}{2}\right)^{2 m+\frac{v}{k}}\left(\frac{y p(x)}{2}\right)^{2 s+\frac{\mu}{k}}}{\Gamma_{s, k}[v+m k+k][m]_{s^{k}} !\left[s+\frac{\mu}{k}\right]_{s^{k}} ![s]_{s^{k}} !}
\end{aligned}
$$

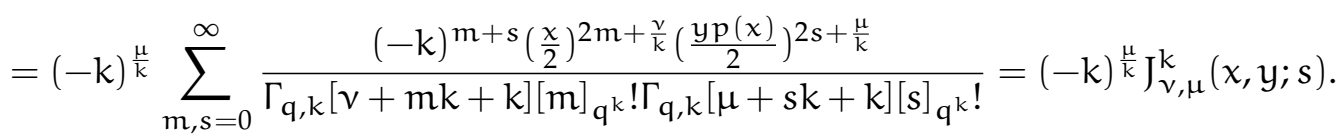

Theorem 4.11. The (s, $\mathrm{k})$-Bessel function in two variables is s-analogy of $\mathrm{k}$-Bessel function in two variables,

$$
\lim _{s \rightarrow 1} J_{v, \mu}^{k}[(1-s) x,(1-s) y ; s]=J_{v, \mu}^{k}(x, y),
$$

where $v, \mu$ are non negative integers, $\mathrm{k}$ is any positive real number and $0<\mathrm{s}<1$.

Proof. Consider the $(\mathrm{s}, \mathrm{k})$-Bessel function is in two variables

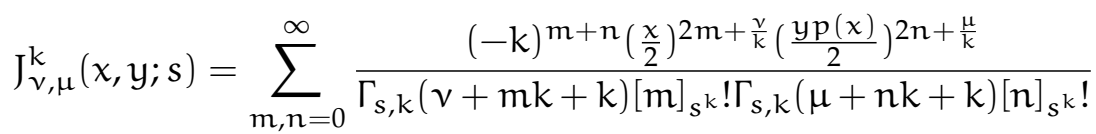

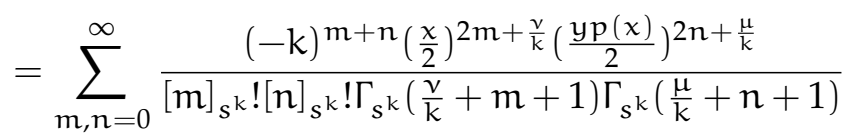

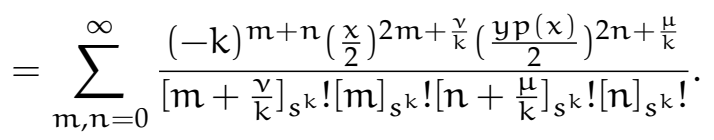

By taking left hand side of the equation (4.19) and using the equation (2.2), we have

$$
\begin{aligned}
& \lim _{s \rightarrow 1} J_{v, \mu}^{k}((1-s) x,(1-s) y ; s) \\
& \quad=\lim _{s \rightarrow 1} \sum_{m, n=0}^{\infty} \frac{(-k)^{m+n}(1-s)^{m+\frac{v}{k}}(1-s)^{m}(1-s)^{n+\frac{\mu}{k}}(1-s)^{n}\left(\frac{x}{2}\right)^{2 m+\frac{v}{k}\left(\frac{y p(x)}{2}\right)^{2 n+\frac{\mu}{k}}}}{\left(s^{k} ; s^{k}\right)_{m+\frac{v}{k}}\left(s^{k} ; s^{k}\right)_{m}\left(s^{k} ; s^{k}\right)_{n+\frac{\mu}{k}}\left(s^{k} ; s^{k}\right)_{n}} .
\end{aligned}
$$

Gaspor [7] has given the relation

$$
((s ; s))_{n+r}=(s ; s)_{r}\left(s^{r+1} ; s\right)_{n}
$$

By using the relation defined in the equation (4.20),

$$
\begin{aligned}
& =\lim _{s \rightarrow 1} \frac{(1-s)^{\frac{v}{k}}(1-s)^{\frac{\mu}{k}}}{\left(s^{k} ; s^{k}\right)_{\frac{v}{k}}\left(s^{k} ; s^{k}\right)_{\frac{\mu}{k}}} \sum_{m, n=0}^{\infty} \frac{(-k)^{m+n}(1-s)^{2 m}(1-s)^{2 n}\left(\frac{x}{2}\right)^{2 m+\frac{v}{k}\left(\frac{y p(x)}{2}\right)^{2 n+\frac{\mu}{k}}}}{\left(\left(s^{k}\right)^{\frac{v}{k}+1} ; s^{k}\right)_{m}\left(s^{k} ; s^{k}\right)_{m}\left(\left(s^{k}\right)^{\frac{\mu}{k}+1} ; s^{k}\right)_{n}\left(s^{k} ; s^{k}\right)_{n}} \\
& =\frac{1}{\Gamma\left(\frac{v}{k}+1\right) \Gamma\left(\frac{\mu}{k}+1\right)} \sum_{m, n=0}^{\infty} \frac{(-1)^{m+n}\left(\frac{x}{2}\right)^{2 m+\frac{v}{k}}\left(\frac{y p(x)}{2}\right)^{2 n+\frac{\mu}{k}}}{k^{\frac{v+\mu}{k}+m+n}\left(\Gamma(1)(1)_{m}\right)\left(\frac{v}{k}+1\right)_{m}\left(\frac{\mu}{k}+1\right)_{n}\left(\Gamma(1)(1)_{n}\right)} \\
& =\sum_{m, n=0}^{\infty} \frac{(-1)^{m+n}\left(\frac{x}{2}\right)^{2 m+\frac{v}{k}}\left(\frac{y p(x)}{2}\right)^{2 n+\frac{\mu}{k}}}{m ! n ! \Gamma_{k}(v+m k+k) \Gamma_{k}(\mu+n k+k)}=J_{v, \mu}^{k}(x, y) .
\end{aligned}
$$

\section{Conclusion}

In our work, the two parameter deformation of classical Bessel function is introduced. We discussed some important relations between k-Bessel function and simple Bessel function in two variables. Also, 
we developed the generating functions which satisfies the k-Bessel function and $(s, k)$-Bessel function in two variables. Moreover, we established a result in which $(s, k)$-Bessel function is s-analogy of k-Bessel function. If $k=1$, generalized $(s, k)$-Bessel function reduces to $s$-Bessel function in two variables. By taking $s=1$ in $(s, k)$-Bessel function, we get $k$-Bessel function in two variables. For $s=1, k=1$, the generalized $(s, k)$-Bessel function reduces to simple classical Bessel function.

\section{References}

[1] P. Agarwal, M. Jleli, M. Tomar, Certain Hermite-Hadamard type inequalities via generalized k-fractional integrals, J. Inequal. Appl., 2017 (2017), 10 pages. 1

[2] R. Diaz, E. Pariguan, On hypergeometric functions and pochhammer k-symbol, Divulg. Mat., 15 (2007), 179-192. 1

[3] R. Diaz, C. Teruel, q, k-Generalized Gamma and Beta Functions, J. Nonlinear Math. Phys., 12 (2008), 118-134. 1, 2.2

[4] H. Exton, q-Hypergeometric Functions and Applications, Halstead Press, New York, (1983). 2.1

[5] G. Farid, G.-M. Habibullah, An extension of Hadamard fractional integral, Int. J. Math. Anal., 9 (2015), 471-482. 1

[6] G. Farid, A. Ur Rehman, M. Zahra, On Hadamard-type inequalities for k-fractional integrals, Konuralp J. Math., 4 (2016), 79-86. 1

[7] G. Gasper, M. Rahman, Basic Hypergeometric Series, Cambridge University Press, Cambridge, (1990). 4

[8] K. S. Gehlot, Differential Equation of k-Bessel's Function and its Properties, Nonlinear Anal. Differ. Equ., 2 (2014), 61-67.

[9] C.-J. Huang, G. Rahman, K. S. Nisar, A. Ghaffar, F. Qi, Some inequalities of the Hermite-Hadamard type for k-fractional conformable integrals, Aust. J. Math. Anal. Appl., 16 (2019), 9 pages. 1

[10] S. Iqbal, S. Mubeen, M. Tomar, On Hadamard k-fractional integrals, J. Fract. Calc. Appl., 9 (2018), 255-267. 1

[11] F. H. Jakson, The Application of Basic Numbers to Bessel's and Legendre's Functions, Proc. London Math. Soc. (2), 2 (1905), 192-220.

[12] C. G. Kokologiannaki, Properties and inequalities of generalized k-gamma, beta and zeta functions, Int. J. Contemp. Math. Sci., 5 (2010), 653-660. 1

[13] V. Krasniqi, A limit for the k-gamma and k-beta function, Int. Math. Forum, 5 (2010), 1613-1617. 1

[14] S. Li, Y. Dong, k-Hypergeometric series solutions to one type of non-homogeneous k-hypergeometric equations, Symmetry, 2019 (2019), 11 pages 1

[15] M. Mansour, Determining the k-generalized gamma function $\Gamma_{\mathrm{k}}(\mathrm{x})$ by functional equations, Int. J. Contemp. Math. Sci., 4 (2009), 1037-1042. 1

[16] F. Merovci, Power product inequalities for the $\Gamma_{k}$ function, Int. J. Math. Anal. (Ruse), 4 (2010), 1007-1012. 1

[17] S. Mubeen, Solution of some integral equations involving confluen k-hypergeometric functions, Appl. Math., 4 (2013), 9-11. 1

[18] S. Mubeen, G. M. Habibullah, An integral representation of some k-hypergeometric functions, Int. Math. Forum, 7 (2012), 203-207. 1

[19] S. Mubeen, S. Iqbal, Grüss type integral inequalities for generalized Riemann-Liouville k-fractional integrals, J. Inequal. Appl., 2016 (2016), 13 pages. 1

[20] S. Mubeen, S. Iqbal, Z. Iqbal, On Ostrowski type inequalities for generalized $k$-fractional integrals, J. Inequal. Spec. Funct., 8 (2017), 107-118. 1

[21] S. Mubeen, C. G. Kokologiannaki, G. Rahman, M. Arshad, Z. Iqbal, Properties of generalized hypergeometric kfunctions via k-fractional calculus, Far East J. Appl. Math., 96 (2017), 351-372. 1

[22] S. Mubeen, M. Naz, A. Rehman, G. Rahman, Solutions of k-hypergeometric differential equations, J. Appl. Math., 2014 (2014), 13 pages. 1

[23] S. Mubeen, A. Rehman, A Note on k-Gamma function and Pochhammer k-symbol, J. Inf. Math. Sci., 6 (2014), $93-107$. 1

[24] K. S. Nisar, G. Rahman, J. S. Choi, S. Mubeen, M. Arshad, Certain Gronwall type inequalities associated with RiemannLiouville k-and hadamard k-fractional derivatives and their applications, East Asian Math. J., 34 (2018), 249-263. 1

[25] F. Qi, S. Habib, S. Mubeen, M. N. Naeem, Generalized k-fractional conformable integrals and related inequalities, AIMS Math., 4 (2019), 343-358. 1

[26] F. Qi, G. Rahman, S. M. Hussain, W. S. Du, K. S. Nisar, Some inequalities of Čhebyšev Type for conformable k-Fractional integral operators, Symmetry, 2018 (2018), 16 pages. 1

[27] G. Rahman, M. Arshad, S. Mubeen, Some results on generalized hypergeometric k-functions, Bull. Math. Anal. Appl., 8 (2016), 66-77. 1

[28] G. Rahman, K. S. Nisar, A. Ghaffar, F. Qi, Some inequalities of the Grüss type for conformable k-fractional integral operators, Rev. R. Acad. Cienc. Exactas Fís. Nat. Ser. A Mat. RACSAM, 114 (2020), 9 pages. 1

[29] G. Rahman, S. Mubeen, K. S. Nisar, On generalized k-fractional derivative operator, AIMS Math., 5 (2019), $1936-1945$. 1

[30] E. D. Rainville, Special functions, Macmillan Co., New York, (1960). 4 
[31] M. Samraiz, E. Set, M. Hasnain, G. Rahman, On an extension of Hadamard fractional derivative, J. Inequal. Appl., 2019 (2019), 15 pages. 1

[32] E. Set, M. A. Noor, M. U. Awan, A. Gözpinar, Generalized Hermite-Hadamard type inequalities involving fractional integral operators, J. Inequal. Appl., 2017 (2017), 10 pages. 1

[33] R. F. Swarthow, An Addition Theorem and Some Product Formulas for the Hahn-Exton q-Bessel Functions, Canad. J. Math., 44 (1992), 867-879. 1

[34] A. Tanguria, R. Sharma, Advanced q-Bessel Function of Two Variables, Int. J. Sci. Eng. Res., 5 (2014), 2229-5518. 2.3

[35] M. Tomar, S. Mubeen, J. S. Choi, Certain inequalities associated with Hadamard k-fractional integral operators, J. Inequal. Appl., 2016 (2016), 14 pages. 1 